\title{
Bacterial Contamination of Circuit Inner Surfaces After High-Flow Oxygen Therapy
}

\author{
Mutsuo Onodera, Emiko Nakataki, Nobuto Nakanishi, Taiga Itagaki, Masami Sato, \\ Jun Oto, and Masaji Nishimura
}

\begin{abstract}
BACKGROUND: During high-flow oxygen therapy, heated humidified gas is delivered at high flow. Although the warmth and humidity of this gas facilitates mucociliary function in the lower airway, warm and humid conditions also promote bacterial growth. Bacterial contamination of high-flow oxygen therapy circuits has not been well investigated. We examined the incidence of bacterial contamination in high-flow oxygen therapy circuits. METHODS: This was a prospective observational study in a university hospital 10-bed general ICU. After final high-flow oxygen therapy discontinuation, samples were obtained from the interface and the chamber ends of the circuit. Initially, a half circumference of each inner surface was swabbed, after which the whole circuit was left in the ICU at room temperature for $6 \mathrm{~h}$. The samples were then, in the same way, taken from the previously unswabbed arcs of the end inner surfaces. All the samples were sent to a biology laboratory and cultured. RESULTS: In all, 118 samples were collected from 31 circuits. The median duration of high-flow oxygen therapy was $48 \mathrm{~h}$ (interquartile range, 26-96 h). Of 31 circuits, contamination occurred in 5 circuits $(16.1 \%$ [95\% CI 5.5-33.7\%]). CONCLUSIONS: Bacterial contamination of inner surfaces of the circuit after high-flow oxygen therapy was relatively high. Key words: hospital-acquired pneumonia; interface; heated humidifier. [Respir Care 2019;64(5):545-549. (C) 2019 Daedalus Enterprises]
\end{abstract}

\section{Introduction}

Mechanical ventilation with an endotracheal tube has been identified as a primary risk factor for ventilator-associated pneumonia (VAP). ${ }^{1,2}$ Despite current efforts to control VAP and hospital-acquired pneumonia, ${ }^{3}$ even in patients who have not received mechanical ventilation, it remains a matter of concern. Many studies have focused on managing risk factors to prevent VAP and a few more

Drs Onodera, Nakataki, Nakanishi, Itagaki, and Nishimura are affiliated with the Emergency and Critical Care Medicine, Tokushima University Graduate School, Tokushima, Japan. Ms Masami Sato is affiliated with the Department of Laboratory Medicine, Tokushima University Hospital, Tokushima, Japan. Dr Oto is affiliated with the Emergency and Disaster Medicine, Tokushima University Hospital, Tokushima, Japan.

The authors have disclosed no conflicts of interest.

Correspondence: Masaji Nishimura MD PhD, Emergency and Critical Care Medicine, Tokushima University Graduate School, 3-18-15 Kuramoto, Tokushima, Japan 770-8503. E-mail: nmasaji@ tokushima-u.ac.jp.

DOI: $10.4187 /$ respcare.06299 recent publications have addressed the epidemiology of hospital-acquired pneumonia in subjects who were not mechanically ventilated. ${ }^{4}$ Both hospital-acquired pneumonia and VAP have a statistically significant impact on morbidity and mortality. 4

Organisms from the gastrointestinal tracts of patients may also cause hospital-acquired pneumonia. ${ }^{5,6}$ During mechanical ventilation, these microbes can rapidly colonize the stomach, oropharynx, lower airways, and endotracheal tube. Circuit colonization occurs rapidly when the source originates from the patient, ${ }^{7}$ and it is the presence of condensate that allows the bacteria to thrive. ${ }^{8,9}$ High-flow oxygen therapy delivers, usually through a large-bore nasal cannula, heated humidified gas at high flow. ${ }^{10}$ Patients who receive high-flow oxygen therapy are less likely to undergo tracheal intubation than those who receive conventional oxygen therapy ${ }^{11,12}$ or noninvasive ventilation. ${ }^{13}$ The warmth and humidity of delivered gas in high-flow oxygen therapy facilitates mucociliary function in the respiratory tract.

Warm humid gas, however, also creates conditions that favor bacterial growth, which increases the risk of bacterial contamination of high-flow oxygen therapy circuits. In 


\section{Bacterial Contamination After High-Flow Oxygen Therapy}

addition, when the ambient temperature is low, the amount of condensation in the circuit increases. ${ }^{14}$ However, gas flow is different between invasive mechanical ventilation and high-flow oxygen therapy. High-flow oxygen therapy is an open system, and flow is uni-directional. In a patient who is intubated, microorganisms that originate from patients can spread into a ventilator circuit with ease; however, it is unclear if microorganisms that originate from patients spread into a high-flow oxygen therapy circuit and can cause hospital-acquired pneumonia.

Although routine circuit changes are not recommended in mechanical ventilation, ${ }^{15,16}$ contamination of breathing circuits has been reported as a source of infection. ${ }^{17,18}$ High-flow oxygen therapy is sometimes applied intermittently, depending on patient condition or tolerance. If a patient resumes high-flow oxygen therapy within several hours, then the same circuit may be used, although this may also accelerate contamination. Because there is little published information about contamination of high-flow oxygen therapy circuits, we designed a study to investigate the incidence of bacterial contamination of the circuit after high-flow oxygen therapy. We also investigated whether the incidence of contamination increased when circuits were left at the bedside.

\section{Methods}

This was a prospective observational study in a university hospital, 10-bed, general ICU. The study protocol was approved by the ethical committee of Tokushima University Hospital (no. 2693). Because high-flow oxygen therapy is routinely used in our ICU and because this was a purely observational study, informed consent was obtained by an opt-out process. The outline of the study was posted on the bulletin board in the ICU. The subjects and their delegates were able to refuse to participate in the study. Regardless of age, each subject in the ICU who received high-flow oxygen therapy for $>6 \mathrm{~h}$ was enrolled. For adults, high-flow oxygen therapy was delivered through an AF2310 chamber and Flextube single heated wire circuit (Intersurgical, Berkshire, United Kingdom), and, for children, through an MR290 chamber and RT330 Optiflow tubing kit for infants (Fisher and Paykel Healthcare, Auckland, New Zealand). The interface was selected, depending on the patient's age and the presence or absence of tracheostomy, from the Optiflow lines of adult and pediatric nasal cannulas or Optiflow tracheostomy direct connections (Fisher and Paykel Healthcare). In routine admission screening, nasal swab samples were taken from our patients in the ICU.

After final discontinuation of high-flow oxygen therapy, culture samples were obtained. To confirm the bacterial origin, we obtained culture samples from the interface and chamber end of the circuit (Fig. 1). Initially, half circum-

\section{QUICK LOOK}

\section{Current knowledge}

High-flow oxygen therapy delivers heated and humidified medical gas to a patient. Warm and humidified inspiratory gas facilitates mucociliary function. However, condensation in high-flow oxygen therapy circuits is common and bacterial contamination cannot be completely avoided.

\section{What this paper contributes to our knowledge}

Bacterial contamination of the inner surface of circuits was investigated just after termination of high-flow oxygen therapy and $6 \mathrm{~h}$ later. Five of 31 circuits revealed bacterial contamination, which was higher than expected. Six hours of exposure to ambient conditions did not increase the contamination rate. Bacterial contamination of circuit inner surfaces was relatively high. A short interruption of high-flow oxygen therapy did not affect bacterial growth.

ferences of the inner surfaces were swabbed with separate Seedswabs (Eiken Chemical Co., Tochigi, Japan). In our daily practice, high-flow oxygen therapy systems are left at the bedside for, at most, $6 \mathrm{~h}$, and we reused the same systems when high-flow oxygen therapy was resumed within $6 \mathrm{~h}$. When high-flow oxygen therapy was not resumed within $6 \mathrm{~h}$, we discarded the circuit. Samples were obtained by the same method, taken from the previously unswabbed arcs of the end inner surfaces after the entire circuit was left in the ICU at room temperature for $6 \mathrm{~h}$. Two samples were taken just after discontinuation of highflow oxygen therapy and two more samples were taken $6 \mathrm{~h}$ later. All samples were sent to the hospital microbiology laboratory for culturing.

\section{Data Collection}

Nasal swab culture results and other subject data, including the indication for high-flow oxygen therapy, the duration of high-flow oxygen therapy, type of circuit, and type of interface, were collected from the medical records. The incidence of bacterial contamination of the circuit was the primary outcome; secondary outcomes included the differences in contamination at each sampling site, sampling time, type of circuit, and type of interface. The association between contamination and duration of high-flow oxygen therapy was also investigated.

\section{Statistics}

Dichotomous variables were compared by using the Fisher exact test. The Wilcoxon rank-sum test was applied 


\section{Bacterial Contamination After High-Flow Oxygen Therapy}

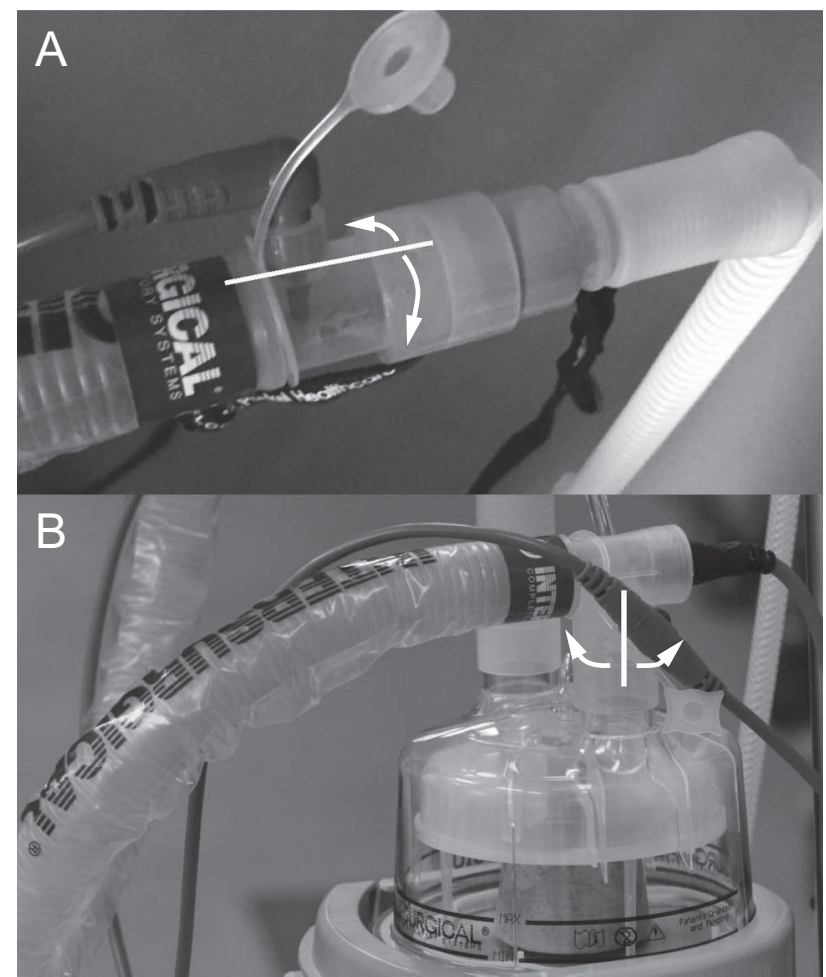

Fig. 1. Locations of microbiological sampling. A: Patient interface end. B: Chamber end. Half of the inner circumference was swabbed immediately after high-flow oxygen therapy and the remaining inner arc was swabbed $6 \mathrm{~h}$ after high-flow oxygen therapy.

to compare the duration of high-flow oxygen therapy between the groups with and without contamination. All analyses were performed with R software version 3.4.2 (http:// www.r-project.org. Accessed October 10, 2017). The level of significance was set at 0.05 .

\section{Results}

Thirty-one subjects were enrolled: 15 females and 16 males. The median age was 73 y (range, $0-93$ y). Twenty six were adults, and 5 were pediatric patients. Nasal cannulas were used for 24 subjects, and tracheal interfaces were used for the remaining 7 subjects. The indications for high-flow oxygen therapy were postextubation $(n=25)$, respiratory failure $(n=4)$, and cardiac failure $(n=2)$. The median (interquartile range) duration of high-flow oxygen therapy was $48 \mathrm{~h}$ (26-96 h). No damage or soiling of the high-flow oxygen therapy circuit was visibly apparent after use. We could not take samples from 3 circuits after $6 \mathrm{~h}$ of high-flow oxygen therapy because circuits were discarded before the second sampling, which resulted in a total of 118 samples. When the culture was positive, regardless of the number of colonies, we defined it as contaminated.
Of 31 circuits, contamination occurred in 5 (16.1\% [95\% CI 5.5-33.7\%]). All contaminated circuits were from adults, including 4 nasal cannula and one tracheal interface. Just after discontinuation of high-flow oxygen therapy, 5 samples exhibited positive cultures, including 4 from the interface end and one from the humidifier chamber end. At $6 \mathrm{~h}$ after high-flow oxygen therapy, 3 samples from the interfaces exhibited positive cultures. These were predominantly, 7 of 8 , from the interface end. Five species of bacteria were isolated (Table 1). However, the strains were different between the samples from circuits and those from nasal swabs at ICU admission. No differences were found in the contamination rate among the types of circuits $(19.2 \%$ in adults vs $0 \%$ in pediatric subjects, $P=.56)$ and the types of interface (16.7\% in nasal cannula vs $14.3 \%$ in the tracheal interface, $P>.99$ ). When contamination occurred, the median (interquartile range) duration of highflow oxygen therapy was $94 \mathrm{~h}(24-96 \mathrm{~h})$. The duration of high-flow oxygen therapy did not differ between circuits with and without contamination $(P=.85)$.

\section{Discussion}

To our knowledge, this study was the first investigation of bacterial contamination of circuits after high-flow oxygen therapy. We found the rate of contamination of the inner surface of the circuit after high-flow oxygen therapy to be $16.1 \%$. Contamination mainly occurred at the interface end. Because high-flow oxygen therapy entails a continuous unidirectional gas flow, we anticipated that contamination in high-flow oxygen therapy circuits would occur less frequently than in anesthetic circuits, where, depending on sampling techniques, the rate of contamination has been found to be from 3.3\% to 54\%. ${ }^{19-21}$ Analysis of our findings indicated that the contamination rate for high-flow oxygen therapy circuits may be as high as for anesthetic breathing circuits.

Because contamination was predominantly found at the interface end, it seemed that the source of bacteria was mostly from the patients. This was similar to the general findings for VAP and hospital-acquired pneumonia, which are mainly caused by organisms found in the gastrointestinal tracts of patients. During mechanical ventilation, circuit colonization from a patient source may occur rapidly. The presence of condensate, in particular, allows the bacteria to thrive. Heat and moisture exchangers decrease the volume of condensate in the circuit and possibly reduce rates of VAP. ${ }^{4,8}$ High-flow oxygen therapy delivers a 1-way high flow of warm humidified gas, and our finding that $16.1 \%$ of the systems were contaminated refuted our expectations. However, patients do exhale against the flow of gas delivered via high-flow oxygen therapy devices, and bacteria from patients may reach the interface. If organisms make it to the interface, then the temperature and 


\section{Bacterial Contamination After High-Flow Oxygen Therapy}

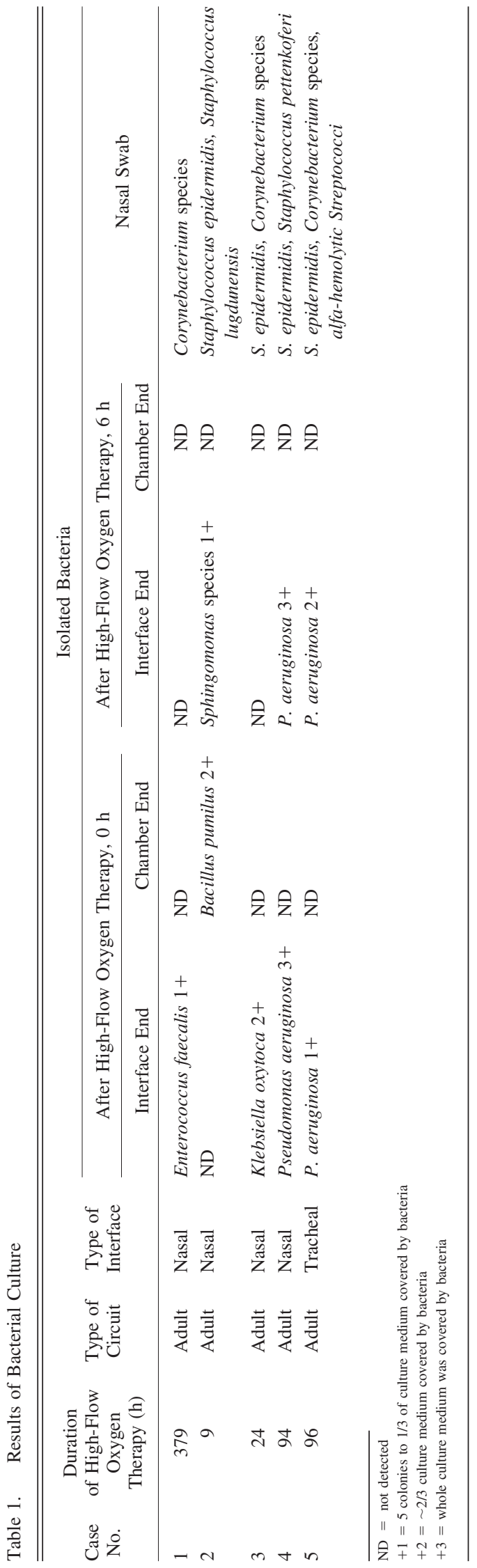

humidity are suitable for proliferation. In our ICU, we use high-flow oxygen therapy circuits fitted with heating wires, which greatly reduce condensation. Condensation is common in non-heated interface circuits.

In case 2, Bacillus pumilus was cultured from a sample taken at the chamber end. High-flow oxygen therapy systems in our ICU are prepared by medical technicians who follow sterile procedures. Given that only a single sample revealed contamination and because a negative sample from the same site was obtained $6 \mathrm{~h}$ later, the single positive result for this particular species may have been due to sampling contamination.

We found no difference in the rate of contamination between samples taken immediately after high-flow oxygen therapy and $6 \mathrm{~h}$ later. Several hours' interruption of high-flow oxygen therapy did not increase bacterial contamination. In cases 1 and 3, bacteria were not cultured from samples $6 \mathrm{~h}$ after high-flow oxygen therapy. This may be due to a problem with sampling because we swabbed the half of circumference at interface end and the chamber end. If we would have swabbed the whole circumference, sample cultures may have been positive at both times. Usually microorganisms originate from patients, and, in patients who are intubated, microorganisms proliferate within the ventilator circuit. Microorganisms could grow in condensates in the present system; however, the highflow oxygen therapy circuits used did not have a water trap. We had to cut the circuit to take samples from the middle, and this method may underestimate the contamination rate.

Another possibility is that, in our normal practice, we hook the interface from a pole when the device is not in use. As a result, condensate drains from the circuit and the interface dries. Although the contamination rate did not vary with sampling time, high-flow oxygen therapy circuits are intended for single use, and the finding cannot be taken to justify the use of the same circuit for the same patient after high-flow oxygen therapy has been suspended for several hours. The duration of high-flow oxygen therapy was similar for cases of contamination and non-contamination. This result was consistent with previous comparisons of 1-d and 7-d use of anesthetic circuits. ${ }^{19-21}$

Our study had several limitations. First, because none of the subjects showed any symptoms of hospital-acquired pneumonia, the clinical impact was unclear: contamination of high-flow oxygen therapy circuits does not necessarily lead to hospital-acquired pneumonia. Moreover, the sample size was too small to come to any generalizable conclusions. Even so, our results were consistent with similar studies of anesthetic breathing circuits. In 3 circuits that yielded positive samples immediately after high-flow oxygen therapy, no contamination was revealed from samples taken $6 \mathrm{~h}$ later. Bacteria may have been present but in such small numbers that none survived to culture or the 


\section{Bacterial Contamination After High-Flow Oxygen Therapy}

distribution of contaminating organisms may have been patchy; if so, the contamination rate after $6 \mathrm{~h}$ may be underestimated. We only swabbed 2 sites to take samples for bacterial cultures. Bacteria could be in condensate within the circuit, and we did not take condensate for cultures. It was almost impossible to get condensate with the sterile procedure, because the high-flow oxygen therapy circuits did not contain a water trap. Also, microbiological samples were obtained from only 3 locations, either end of the circuit and from the nose; consequently, the origins of the detected bacteria are unclear.

\section{Conclusions}

Bacterial contamination of the inner surface of the circuit after high-flow oxygen therapy was relatively high. Short interruption of high-flow oxygen therapy did not affect bacterial growth. Our study highlights the potential risk of bacterial contamination of high-flow oxygen therapy circuits; however, many questions remain to be clarified. Further studies should explore potential risk factors for circuit contamination, the interaction between patient colonization and system colonization, the role of highflow oxygen therapy duration, incidence of pneumonia, and ICU length of stay.

\section{REFERENCES}

1. Branson RD. The ventilator circuit and ventilator-associated pneumonia. Respir Care 2005;50(6):774-785; discussion 785-787.

2. Phu VD, Nadjm B, Duy NHA, Co DX, Mai NTH, Trinh DT, et al. Ventilator-associated respiratory infection in a resource-restricted setting: impact and etiology. J Intensive Care 2017;5:69.

3. Cameron JI, Chu LM, Matte A, Tomlinson G, Chan L, Thomas C, et al.; RECOVER Program Investigators (Phase 1: towards RECOVER), Canadian Critical Care Trials Group. One-year outcomes in caregivers of critically ill patients. N Engl J Med 2016; 374(19):1831-1841.

4. Stergiopoulos S, Calvert SB, Brown CA, Awatin J, Tenaerts P, Holland TL, et al. Cost drivers of a hospital-acquired bacterial pneumonia and ventilator-associated bacterial pneumonia phase 3 clinical trial. Clinical infectious diseases. Clin Infect Dis 2018;66(1):72-80.

5. Fortaleza CM, Abati PA, Batista MR, Dias A. Risk factors for hospital-acquired pneumonia in nonventilated adults. Braz J Infect Dis 2009;13(4):284-288.

6. Fortaleza CM, Figueiredo LC, Beraldo CC, Melo EC, Póla PM, Aragão VD. Risk factors of oropharyngeal carriage of Pseudomonas aeruginosa among patients from a Medical-Surgical Intensive Care Unit. Braz J Infect Dis 2009;13(3):173-176.

7. Craven DE, Connolly MG Jr, Lichtenberg DA, Primeau PJ, McCabe WR. Contamination of mechanical ventilators with tubing changes every 24 or 48 hours. N Engl J Med 1982;306(25):1505-1509.
8. Kirton OC, DeHaven B, Morgan J, Morejon O, Civetta J. A prospective, randomized comparison of an in-line heat moisture exchange filter and heated wire humidifiers: rates of ventilatorassociated early-onset (community-acquired) or late-onset (hospital-acquired) pneumonia and incidence of endotracheal tube occlusion. Chest 1997;112(4):1055-1059.

9. Roustan JP, Kienlen J, Aubas P, Aubas S, du Cailar J. Comparison of hydrophobic heat and moisture exchangers with heated humidifier during prolonged mechanical ventilation. Intensive Care Med 1992; 18(2):97-100.

10. Nishimura M. High-flow nasal cannula oxygen therapy in adults: physiological benefits, indication, clinical benefits, and adverse effects. Respir Care 2016;61(4):529-541.

11. Ni YN, Luo J, Yu H, Liu D, Ni Z, Cheng J, et al. Can high-flow nasal cannula reduce the rate of endotracheal intubation in adult patients with acute respiratory failure compared with conventional oxygen therapy and noninvasive positive pressure ventilation?: A systematic review and meta-analysis. Chest 2017;151(4):764-775.

12. Zhao H, Wang H, Sun F, Lyu S, An Y. High-flow nasal cannula oxygen therapy is superior to conventional oxygen therapy but not to noninvasive mechanical ventilation on intubation rate: a systematic review and meta-analysis. Crit Care 2017;21(1):184.

13. Frat JP, Brugiere B, Ragot S, Chatellier D, Veinstein A, Goudet V et al. Sequential application of oxygen therapy via high-flow nasal cannula and noninvasive ventilation in acute respiratory failure: an observational pilot study. Respir Care 2015;60(2):170-178.

14. Chikata Y, Unai K, Izawa M, Okuda N, Oto J, Nishimura M. Inspiratory tube condensation during high-flow nasal cannula therapy: a bench study. Respir Care 2016;61(3):300-305.

15. Hess DR, Kallstrom TJ, Mottram CD, Myers TR, Sorenson HM, Vines DL. Care of the ventilator circuit and its relation to ventilatorassociated pneumonia. Respir Care 2003;48(9):869-879.

16. Tablan OC, Anderson LJ, Besser R, Bridges C, Hajjeh R, Healthcare Infection Control Practices Advisory Committee. Guidelines for preventing health-care-associated pneumonia, 2003: recommendations of CDC and the Healthcare Infection Control Practices Advisory Committee. MMWR Recomm Rep 2004;53(RR-3):1-36.

17. Gorman LJ, Sanai L, Notman AW, Grant IS, Masterton RG. Cross infection in an intensive care unit by Klebsiella pneumoniae from ventilator condensate. J Hosp Infect 1993;23(1):27-34.

18. Schulz-Stübner S, Kniehl E. Transmission of extended-spectrum beta-lactamase Klebsiella oxytoca via the breathing circuit of a transport ventilator: root cause analysis and infection control recommendations. Infect Control Hosp Epidemiol 2011;32(8):828-829.

19. Dubler S, Zimmermann S, Fischer M, Schnitzler P, Bruckner T, Weigand MA, et al. Bacterial and viral contamination of breathing circuits after extended use - an aspect of patient safety? Acta Anaesthesiol Scand 2016;60(9):1251-1260.

20. McGain F, Algie CM, O'Toole J, Lim TF, Mohebbi M, Story DA, Leder K. The microbiological and sustainability effects of washing anaesthesia breathing circuits less frequently. Anaesthesia 2014;69(4): 337-342.

21. Hartmann D, Jung M, Neubert TR, Susin C, Nonnenmacher C, Mutters R. Microbiological risk of anaesthetic breathing circuits after extended use. Acta Anaesthesiol Scand 2008;52(3):432-436. 